\title{
Indicadores trazadores de gestión hospitalaria. Revisión, análisis y propuesta
}

\author{
CAROLINA TORRES(1), NELLY ALVARADO'(1,2)
}

\section{RESUMEN}

Las instituciones prestadoras de servicios de atención médica han debido asumir una postura de adaptación continua y permanente a los cambios, ya sea frente a sus clientes, al recambio tecnológico y a las variaciones de la práctica clínica, como a su propia sustentabilidad financiera. En consecuencia, la toma de decisiones se ha complejizado con el aumento de la cantidad, variedad y tipos de resultados de las intervenciones sanitarias. Al mismo tiempo se ha creado un infinito volumen de mediciones de recursos, procesos, resultados e impacto de tales acciones, sin que exista hoy un consenso absoluto respecto de la utilidad, validez o confiabilidad de tales mediciones. Se realizó una revisión conceptual de definiciones y características de indicadores en salud (bibliográfica y entrevistas), una investigación bibliográfica de experiencias nacionales e internacionales, y una discusión y definición de dimensiones de indicadores trazadores de gestión para la Clínica Dávila, en el marco de su plan de trabajo del año 2003. Se definieron 5 dimensiones: calidad técnica, efectividad, eficiencia, relación con seguros y relación con usuarios. Para cada una, la revisión de experiencias mostró una distribución de indicadores de 58,6\%, $9,7 \%, 45,2 \%, 3,2 \%$ y 22,6\% respectivamente, mientras que el análisis local presentó una distribución de 37,4\%, 0,0\%, 17,14\%, 22,9\% y 22,9\% respectivamente, el que resultó concordante con la mayor prevalencia de indicadores de calidad técnica. Por otra parte, destacan los indicadores locales de relación con usuarios y seguros, dimensiones poco o nada abordadas en otras experiencias. Finalmente, se realizan sugerencias para una propuesta de trabajo en este ámbito para ser desarrollada al interior de la institución.

Palabras claves: indicadores en salud, gestión en salud, usuarios.

\section{ABSTRACT}

INDICATORS FOR HOSPITAL PLANNING MANAGEMENT. REVISION, ANALYSIS AND PROPOSALS

Institutions delivering medical care services have had to take upon themselves a continuous and permanent position of adapting to changes, be it toward their users, toward technological changes and variations in clinical practice, as well as to their own financial sustenance. In consequence, decision making has become complex with the increase in the quantity, variety and types of results of health interventions. At the same time, an infinite volume of measurings of resources, processes, results and impact of such actions has been created, without the existence,

(1) Escuela de Salud Pública. Facultad de Medicina. Universidad de Chile.

(2) Directora Médica de Calidad y Gestión de Riesgos. Clínica Dávila. 
at present, of an absolute agreement regarding the usefulness, validity or reliability of these measurings. A conceptual revision of health characteristics and indicators (both bibliographic and through interviews), a bibliographic investigation of national and international experiences, and a discussion and definition of dimensions of planning management indicators for the Dávila Clinic, were carried out in the framework of its working plan for the year 2003. Five dimensions for: technical quality, effectiveness, efficiency, insurance relations and user relations, were defined. For each one of these, the revision of experiences showed a distribution of indicators of $58.6 \% .9 .7 \%, 45.2 \%, 3.2 \%$, and $22.6 \%$ respectively, while the local analysis presented a distributiuon of $37.4 \%, 0.0 \%, 17.14 \%, 22.9 \%$, and $22.9 \%$ respectively, which resulted to be concordant with the greater prevalence of technical quality indicators. On the other hand, local indicators of user and insurance relations stand out, dimensions which were only slightly approached or not approached in other experiences. Finally, suggestions for a working proposal in this setting, to be developed within the institution, are being given close attention.

Key words: health indicators, hospital management, hospital users.

\section{INTRODUCCIÓN}

Hace un siglo en Chile, la labor de las instituciones de salud se basaba fundamentalmente en actos de beneficencia. Entonces, la conducción de tales instituciones no significaba mayor preocupación para la sociedad, excepto, seguramente, para los religiosos a cargo y para quienes veían en estas instituciones su única forma de acercarse a una atención digna para esa época.

Sin embargo, con el devenir del tiempo, la progresiva penetración de conceptos económicos, como la eficiencia y la inversión, en el desarrollo de instituciones de bien social han determinado que los directores o gerentes de estos establecimientos asistenciales hayan iniciado un creciente interés por optimizar su quehacer de modo de responder no sólo a las demandas de la población, sino también a las necesidades, las que han ido evolucionando invariablemente hacia la multiplicidad y la complejidad.

Las instituciones prestadoras de servicios de atención médica, por otra parte, han debido asumir una postura de adaptación continua y permanente a los cambios, ya sea para hacer frente a las expectativas -cada vez más exigentes- de sus clientes, al avasallador recambio tecnológico y variaciones de la práctica clínica acorde a los avances científicos, así como a su propia susten tabilidad financiera.

En este contexto, la toma de decisiones en salud se ha complejizado paralelamente al aumento de la cantidad, variedad y tipo de resultados de las intervenciones sanitarias, ya sea en el contexto poblacional como en el de cada paciente. Por esta razón, también, se ha creado un infinito volumen de mediciones de recursos, procesos, resultados e impacto de tales acciones, sin que exista hoy un consenso absoluto respecto de la utilidad, validez o confiabilidad de tales mediciones.

El presente trabajo tiene por objetivo aproximarse al ordenamiento de la creación y utilización de indicadores trazadores para la toma de decisiones en la gestión hospitalaria.

\section{MATERIAL Y MÉTODOS}

El método utilizado en el presente trabajo consideró tres etapas superpuestas:

1. Revisión y análisis conceptual de las definiciones y características de los indicadores utilizados en la gestión de instituciones de salud, que se basó a su vez en dos componentes: la búsqueda bibliográfica de publicaciones científicas y textos de estudio, y la discusión con académicos, generadores y usuarios de este tipo de indicadores. Para la búsqueda bibliográfica se utilizó el servicio disponible en Clínica Dávila (PubMed), de textos de clínicas y 
establecimientos de salud españoles, argentinos, uruguayos y mexicanos, entre otros.

2. Revisión bibliográfica de experiencias nacionales e internacionales en el uso de indicadores trazadores de gestión en instituciones de salud disponibles en la web.

3. Discusión y definición de dimensiones de indicadores trazadores para efectos del presente estudio. Esta tarea fue efectuada en conjunto con la Subgerencia de Control y Gestión de la Clínica Dávila utilizando como insumo básico inicial las definiciones del Plan de Trabajo para el año 2003 de la Unidad de Gestión de Riesgos Clínicos de esta institución.

\section{MARCO CONCEP'TUAL}

Históricamente, la calidad del cuidado de los enfermos ha estado determinada por el juicio particular de los profesionales del área de la salud. Sin embargo, con el progresivo aumento de los costos de la atención de salud asociada a recursos escasos, los gobiernos tanto de países desarrollados como de países en vía de desarrollo, en menor o mayor medida, han iniciado desde hace tiempo la búsqueda de evidencia objetiva que permita la entrega de tales cuidados de manera más efectiva y eficiente, manteniendo o incluso incrementando la calidad de tales acciones!

Un elemento fundamental para satisfacer estos objetivos es, sin duda, la medición y evaluación permanente y sistemática de la prestación de servicios de salud, desde sus componentes (recursos financieros, humanos, de infraestructura y tecnologías, entre otros) hasta sus impactos globales, permitiendo vigilar, controlar, evaluar y -eventualmentemodificar los procesos involucrados en la promoción, protección y recuperación de la salud de las personas y de sus comunidades y entornos.

Por otro lado, la disponibilidad de información de dichos procesos es cada vez mayor, tanto en cantidad como en complejidad. Este volumen de información potencialmente disponible para los tomadores de decisión, hace necesaria una exhaustiva selección de aquellos elementos que ameriten el esfuerzo de ser monitorizados.

En este contexto, la construcción y selección de los indicadores es fundamental. Si entendemos que un indicador es la medición o relación de mediciones que reflejan uno o varios aspectos o etapas del proceso que se analiza, que tiene como objetivo su evaluación para orientar la toma de decisiones ${ }^{2,3,5}$, su definición, elaboración y utilización deben ser lo más precisas posibles. Para lograr tal precisión, es fundamental recordar las características que debe tener un indicador (validez, confiabilidad, especificidad, sensibilidad, relevancia y costo-efectividad) ${ }^{2}$. En consecuencia es comprensible que exista una amplia variedad de indicadores para cada etapa y tipo de proceso productivo en salud, así como para los distintos niveles de decisión, que intenta responder a la variedad de aspectos que un tomador de decisiones requiere considerar. El conflicto aparece ante la imposibilidad de manejar efectivamente tal volumen de indicadores, incluso asumiendo que sólo manejará aquellos indicadores de su nivel en la gestión de su institución. Aparece, entonces, la necesidad de definir un conjunto reducido de indicadores, trazadores o resumen, que se instituyan como elementos centinelas del proceso que se analiza y que permita alertar oportunamente al tomador de decisiones.

Existen en este sentido varias iniciativas, la mayoría en países desarrollados, que orientan la elaboración de estos indicadores trazadores, intentando diferenciar dimensiones o ámbitos de pertinencia, labor siempre compleja, considerando los límites habitualmente difusos de tales espacios.

En la siguiente sección se presentan algunas de las experiencias reportadas en la literatura.

\section{ANÁLISIS DE EXPERIENCIAS}

\section{1). Definición y construcción de dimensiones.}

La clasificación de los indicadores utilizados en experiencias analizadas considera diversos criterios, y auncue existe consistencia en la información que se utiliza en la construcción de indicadores y en el enfoque de éstos, las dimensiones consideradas son múltiples.

Se obtuvo información de algunas experiencias disponibles en formato electrónico, 
donde se explicita la construcción y clasificación de indicadores trazadores para la gestión del proceso productivo en salud, que se resumen y ejemplifican a continuación ${ }^{1,2,3,5,6}$. Entre las experiencias analizadas en este trabajo, se consideraron también como tales la información obtenida del Quality Indicator Project(B) de la Asociación de Hospitales y Sistema de Salud de Maryland (2000) y del programa WinSIG(B) elaborado por la Organización Panamericana de la Salud versión 2001.

Las cuatro experiencias que se presentan fueron las más relevantes para los efectos de alcanzar los objetivos previamente señalados para este estudio.

\section{1). Acute Health Performance Indicators}

(Hospital de Victoria, Australia, 1998):

a. Eficiencia: incluyendo costos de tratamiento por egreso, por tipo de caso, estadía promedio de las 20 GRD (grupo relacionado de diagnósticos) más frecuentes.

b. Productividad: relación del costo de reemplazo de equipos por depreciación vs total de reemplazos de equipos, costos de uso del capital por tipo de caso.

c. Calidad: reingresos por emergencia, infecciones nosocomiales, satisfacción del paciente, cumplimiento de estándares de manejo, proporción de camas acreditadas.

d. Acceso: tiempos de espera para cirugía electiva, tiempo de espera para atención en servicios de urgencia, tiempos de espera en consulta ambulatoria.

2) Strategic performance management system at the Mayo Clinic (Outpacient Operations Groups, Mayo Clinic, Minnesota, 1999).

a. Satisfacción usuaria: índice de cuidados primarios, índice de cuidados de subespecialidades.

b. Productividad y eficiencia clínica: actividades por profesional por día, consultas externas por médico por día.

c. Finanzas: costo por unidad productiva.

d. Operación interna: quejas por 1.000 pacientes, tiempos de espera.

e. Respeto mutuo y diversidad: porcentaje de funcionarios de grupos minoritarios, grado de satisfacción de usuarios internos. f. Evaluación externa: participación de mercado.

g. Compromiso social: contribución de Mayo Clinic a la sociedad.

h. Características de los pacientes: mix de origen geográfico y de grupos de pago.

3) Guía para la construcción de Indicadores de Gestión. (Gobierno de Uruguay, 1999). (Ej.: Función de Provisión de Servicios).

a. Desempeño financiero: Porcentaje de gasto en inversiones, gastos de administración vs gastos totales, Ingresos generados por venta de servicios $v s$ ingresos totales.

b. Eficacia: Demanda cubierta vs demanda potencial.

c. Eficiencia: Costo promedio del servicio.

d. Calidad del servicio: Porcentaje de servicios entregados dentro del plazo máximo, tiempo promedio de espera para la entrega del servicio.

e. Desempeño del RRHH: Horas hombre efectivamente trabajadas $v s$ contratadas, Porcentaje de personal destinado a cometidos sustantivos del total de personal, personal capacitado por año vs total del personal.

4) Measuring hospital performance to improve the quality of care in Europe: A need for clarifying the concepts and defining the main dimensions. (Report on a WHO Workshop, Barcelona, España, Enero de 2003.)

a. Efectividad Clínica: Reingresos, mortalidad, tasa de complicaciones, promedio de estadía, procedimientos basados en evidencia.

b. Percepción del paciente: Tiempos de espera, equidad en acceso, derechos de los pacientes, satisfacción usuaria.

c. Eficiencia productiva: Promedio de estada por patología específica.

d. Seguridad: IIH, caídas, escaras de decúbito.

e. Manejo del staff (RRHH): Recambio, tasa de ausentismo.

f. Responsabilidad comunitaria: Modelo estratégico territorial, legitimidad social (reconocimiento, por parte de la comunidad en la que se inserta, de su contribución al estado sanitario local).

\section{2). Revisión de indicadores trazadores.}

Se obtuvo información de 31 publicaciones 


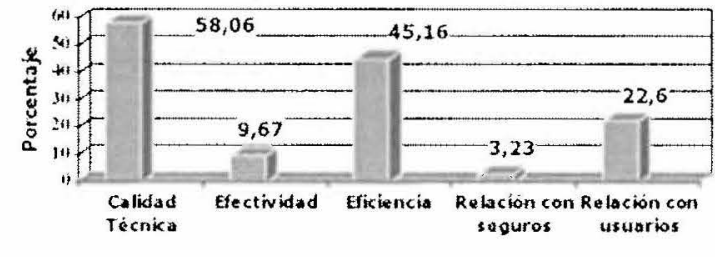

Figura 1. Distribución de publicaciones según dimensiones de indicadores trazadores explicitados.

científicas ${ }^{7-37}$, donde se explicitan diversos indicadores construidos y utilizados en distintas y variadas circunstancias, pero siempre enfocados a mejorar la calidad de la atención de salud. Cabe destacar que la gran mayoría de ellas se enmarca en la difusión de experiencias puntuales de uso de indicadores para demostrar la utilidad de alguna intervención específica.

En el Plan de Trabajo 2003 de la Unidad de Gestión de Riesgos de la Clínica Dávila, se describe como uno de los objetivos específicos: "Constituirse en una Unidad estratégica a través de la elaboración de un sistema de monitoreo de los casos clínicos por medio de información estandarizada y de seguimiento sistemático..." y entre las actividades derivadas de este objetivo, se plantea la elaboración y selección de indicadores trazadores de la gestión clínica en materia de calidad, efectividad, eficiencia, costo/efectividad, relación con seguros, relación con pacientes y sus familiares.

En consecuencia, para clasificar los indicadores, se delimitaron 5 dimensiones, a partir de la información obtenida de la revisión bibliográfica y el trabajo ya desarrollado por la Clínica, a saber: calidad técnica, efectividad, eficiencia, relación con seguros (financiadores) y relación con usuarios (clientes/pacientes).

Al asociar los artículos revisados según las dimensiones a las que corresponden sus indicadores informados, como muestra la Figura 1, se encontró que las publicaciones se concentran en las dimensiones de calidad técnica $(58,06 \%)$ y eficiencia $(45,16 \%)$.

Es importante destacar que la clasificación

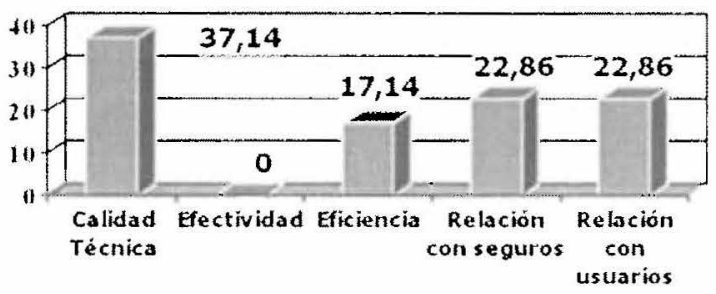

Figura 2. Distribución de indicadores trazadores por dimensión. Clínica Dávila 2003.

de un determinado indicador puede no ser tarea fácil, pues es frecuente que responda a las características definidas en más de alguna de estas categorías, por lo que su ubicación dependerá no sólo de su construcción, sino del uso que se le dé y del contexto en el que se usa.

El análisis por dimensión para la institución Clínica Dávila, es concordante con la revisión bibliográfica en cuanto a la mayor relevancia de los trazadores en calidad técnica, destacando también las dimensiones de relaciones con los clientes y financiadores*.

\section{CONCLUSIONES}

De la información disponible, destaca que la creación de indicadores efectivos para la adecuada gestión de instituciones de salud, independiente del país, es una tarea ardua pero prioritaria. Sin embargo, la amplitud de características de los procesos productivos en salud, su multidisciplinariedad y veloz desarrollo han significado esfuerzos paralelos que, a pesar de los distintos contextos en que se ubican, guardan consistencia al menos en la definición de las dimensiones a considerar.

Pese a lo anterior, es evidente también que hasta ahora, al menos, estos esfuerzos se han concentrado en la elaboración de indicadores que reflejen la calidad técnica y el uso de los recursos, dejando postergada la relación con los distintos clientes (usuarios externos y financiadores) así como la efectividad real de las acciones y servicios de atención.

Finalmente, cabe preguntarse si esta amplia

Para la Clínica se obtuvo un total de 128 indicadores trazadores, excluyéndose de ellos -por no considerarse mazadores-alrededor de 70 indicadores relacionados con la vigilancia y mejoría continua de la calidad que se sighen rutinariamente. 
gama de indicadores está siendo efectivamente utilizada por -y para- los fines que han sido creados, y si aquéllos han sido pertinentemente evaluados en el cumplimiento de su propósito de origen, pues es probable que se acumulen o se abandonen sin haber realizado el cierre del proceso de su implementación, incluso de aquellos que hoy son tradicionales como el índice ocupacional de los hospitales o el rendimiento horario del recursos humano.

Después de la revisión efectuada, nos quedan interrogantes, pues se podría pensar que aquellos indicadores "exitosos" hubiesen sido ya difundidos lo suficiente como para lograr un manejo comparativo de su información, al menos al interior de los países, situación que no pareciera que así sea.

Considerando lo anterior, formulamos una propuesta que se sustenta en dos pilares fundamentales:

1. La revisión y análisis crítico de este artículo, evaluando la validez y utilidad tanto de las dimensiones definidas, como de los tipos de indicadores presentados.

2. La construcción de una herramienta de gestión que permita integrar la información disponible a través de la selección y/o creación de indicadores trazadores, que constituyan un núcleo eje de monitoreo central que hagan sentido a los tomadores de decisión.

Para el logro de tal desafío, es esencial tener algunas consideraciones propias de cualquier proceso de cambio en una institución, y que se pueden resumir en las siguientes:

1. Realizar el proceso de manera explícita y consensuada.

2. Establecer definiciones y objetivos claros del proceso y su producto, explicitando sus usos futuros.

3. Intentar realizar el procedimiento con la participación de todas las personas que tengan directa relación, tanto con la elaboración como en el uso ulterior de la información generada.

4.- Incorporar la visión de los Seguros y los Usuarios de los servicios a través de encuestas y/o entrevistas dirigidas a identificar los elementos relevantes en su percepción de relación con la institución.

5. Aumentar las referencias, buscando ampliar las experiencias en este ámbito, tanto a través de la profundización de la revisión bibliográfica, o a través de visitas a otras instituciones que hayan implementado, o estén en proceso de implementar, herramientas similares para vigilar y mejorar su gestión.

Finalmente, es importante tener presente el desafio de revisar y evaluar en forma rutinaria las mediciones realizadas al interior de los centros prestadores, como forma de mirar la pertinencia de las mismas y su adaptación a los requerimientos de sus variados clientes.

\section{REFERENCIAS}

1.- Victoria's Hospital, Departamento de Recursos Humanos, Australia; Acute Health Performance Indicators; 1998. (http://www.health.vic.gov.au/ clinicalindicators/strategy/parta.htm, consultado el 05/08/03).

2.- Grupo de análisis del Programa Especial de Análisis de Salud (SHA), OPS: Indicadores de Salud: elementos básicos para el análisi de la situación de salud, Bol Epid OPS 22(4), dic 2001.

3.- ARREDONDO A. Indicadores de eficiencia para la asignación de recursos en salud. Rev Méd Chile $1999 ; 127: 856-961$.

4.- The Association of Maryland Hospital \& Health Systems; Quality Indicator Project@ (http:/ www.giproject.org, consultado el 23/10/03).

5.- Gobieno de Uruguay; Guía para la Construcción de Indicadores de Gestión; Feb 1999. (http:// cepre.opp.gub.uy/sitio/areas/SEV/Sev/Metodos/ GuiaIndic.htm, consultado el 21/10/03).

6.- Ministerio de Salud y Acción Social de la Nación. República Argentina; Hospital Público de Autogestión. Marco Conceptual, estrategias e instrumentos operativos. Cap. IX: Fuentes de información hospitalaria, indicadores de gestión para los hospitales públicos. Oct 1996.

7.- AROUZULLAH A M, HENDERSON W G, KIHURF F S, DALEY J. Postoperative mortality and pulmonary complicaction rankings how do they correlate at the hospital level?. Med Care 2003; 41 (8): $979-91$.

8.- GOSDEN T, FORLAND I*, KRISTIANSEN I $S$, SUTTON M, et al. Capitaction, salary, feefor.service and mixed systems of payment. Cochrane Database of Systematic Rewiews, 3, 2003 Citation 27

9. - PARKES J, SHEPPERD S. Discharge planning from hospital to home. Cochrane Database of Systematic 
Rewiews, 3, 2003 Citation 32.

10. - BOWER P, ROWLAND N, MELLOR CLARK J, HEYWOOD $P$, et al. Efectiveness and cost efectiveness of counselling in primary care. Cochrane Database of Systematic Rewiews, 3, 2003 Citation 1.

11.- ZWARENSTEIN M, BRYANT W. Interventions to promote collaboration between nurses and doctors. Cochrane Database of Systematic Rewiews, 3, 2003 Citation 43.

12.- SCHIAVI P, DEVOTO A, ROJO L, MAGARIAN $S$, TOER E. Indicadores de resultados de la gestión hospitalaria. Primer Seminario Nacional sobre Indicadores, República Argentina, julio 2001.

13.- OSÁN M, GRIFONE M. Indicadores de gestión hospitalaria. Programa para aplicar en institución polivalente. Primer Seminario Nacional sobre Indicadores, República Argentina, julio 2001.

14.- GALESIO A. Indicadores de calidad en cuidados intensivos. Primer Seminario Nacional sobre Indicadores, República Argentina, julio 2001.

15.- Mendoza P. Evaluación económica en Salud. Análisis de costos y análisis de costo - efectividad. Rev Méd IPSS 1995; 4(1).

16. CAMPBELL S, BRASPENNING J, HUTCHINSON A, MARSHALL M. Improving the quality of health care. Research methods used in developing and applying quality indicators in primary care. BJM 2003; 326 .

17. MAGEE H, DAVIS L, COULTER A. Public views on healthcare performance indicators and patient choice. JR Soc Med 2003; 96(7): 338-42.

18.- AKINCIF, SINAY T. Perceived access in a managed care environment: determinants satisfaction. Health Serv Manage 2003; I6(2): 85-95.

19.- KAZANDJIAN V, MATTHES N, WICKER K. Are performance indicators generic? The internacional experience of the Quality Indicator Proyect $(\mathrm{B})$. Eval Clin Pract 2003; 9(2): 265-76.

20.- THOMPSON J, PINIDIYA S, RUAN K, MCKINLEY E, et al. Health plan quality-of-care information is undermined by voluntary reporting. Am J Prev Med 2003; 24(1): 62-70.

21.- ROSEN A, RAKOVSKI C, LOVELAND S, ANDERSON J, BERLOWITZ D. Profiling resource use: do different outcomes affect assessments provider efficiency? Am J Manag Care 2002; 8(12): $1105-15$.

22.- ELKIN P, BROWN S, CARTER J, BAUER B, WAHNER-ROEDLER D, et al. Guideline and quality indicators for development, purchase and use of controlled health vocabularies. Int J Med Inf $2002 \mathrm{dec} 18 ; 68(1-3)$ : 175-86.

23. SOLBERG L, LYLES C, SHORE A, LEMKE K, WEINER J. Is quality free? The relationship between cost and quality across 18 providers groups. Am J Manag Care 2002; 8(5): 413-22.
24.- OERMANN M, DILLON $S$, TEMPLIN T. Indicators of quality of care clinics: patient's perpectives. J Health Quał 2000 Nov-Dec; $22(6) ; 9$ 11 ; quiz 12.

25.- CURTRIGHT J, STOLP-SMITH C, EDELL, E. Measuring hospital performance to improve the quality of care in Europe: A need for clarifying and definig the main dimensions. J Health Manag 2000; 45(1): 58-68.

26.- COLLOPY B. Clinical indicators in accreditation: an effective stimulus to improve patient care. Int $J$ Qual Health Care 2000; 12(3): 211-6.

27.- PESKETT M. Clinical indicators and other complications in the recovery romm or postanaesthesic care unit. Anaesthesia. 1999; 54(12): 1143-9.

28.- VAN RIJSWIJK L. Clinical practice guidelines: moving into the $21 \mathrm{st}$ century. Ostomy Wound Manage 1999 Jan; 45(1 A suppl): 47S-53S.

29.- JANSSON A, ISAACSON A, KORNFATL R, LINDHOLIM L. Quality in chiled care. The wiews of mothers and public health nurses. Scan J Caring Sci 1998; 12(4): 195-204.

30.- WU F, LIN J, TSAI W. Decision support system for the analysis of hospital operation indicators. Ann N Y Acad Sci 2002 Dec; 298-305.

31.- HOGGINS T, MCGEE W, STEINGRUB J, RAPOPORT J, LEMESHOW S, TERES R. Early indicators of prolonged intensive care unit stay: impacto of illness severity, physician staffing, and pre-inyensive care unit length of stay. Crit Care Med 2003; 31(1): 45-5I.

32.- DUFFY J. Nosocomial infections: important acute care nursing-sensitive outcomes indicators. AACN Clin Issues 2002; 13(3): 358-66.

33. - RHEW D, GOETZ M, SHEKELLE P. Evaluating qulaity indicators for patients with communityacquired pneumonia. Jt Comm J Qual Improv 2001; 27(11): 575-90.

34. BIAGINI L, MANTEROLA I, SAAVEDRA M, MARTICORENA J, LU1 A. Evaluación de la efectividad de las normas terapéuticas propuestas en el Hospital Clínico Universidad de Chile, para neumonía e infección urinaria. Rev Med Clin Univ Chile 2000; 11(1): 73-82.

35.- Autor no identificado; Patient-driven indicators for quality. Qual Lett Healthe Lead 1998; 10(8): 11-2.

36.- AIBAR C. El logro del value for money en la gestión pública: consideraciones en trono a los indicadores de eficiencia, eficacia y economía; Rev Contab \& Finanç 2003; 32: 99-110.

37.- Measuring hospital performance to improve the quality of care in Europe: a need for clarifing the concepts and defining the main dimensions; Report on a WHO Workshop, Barcelona, España, 10-11 Jan 2003 (http://www.euro.who.int, consultado el 14/11/03). 